\title{
OR11-006 - A mutation in NLRP1A causes autoinflammation
}

\author{
SL Masters", M Gerlic, BT Kile, BA Croker \\ From 7th Congress of International Society of Systemic Auto-Inflammatory Diseases (ISSAID) \\ Lausanne, Switerland. 22-26 May 2013
}

\section{Introduction}

The NLRs (Nucleotide-binding domain and Leucine-rich repeat containing Receptors) are a family of intracellular innate immune receptors involved in host defense. Upon activation, NLRs form large protein complexes called "inflammasomes" that bind and activate Caspase-1, resulting in proteolytic activation of the pro-inflammatory cytokines pro-IL-1 $\beta$ and pro-IL-18 and also induce a Caspase-1-dependent form of cell death known as pyroptosis.

\section{Objectives}

Activating mutations in NLRP3 trigger the inflammasome and cause a spectrum of auto-inflammatory disease. Therefore our objective was to establish if activating mutations in NLRP1 also cause autoinflammatory disease.

\section{Methods}

We performed an $N$-ethyl- $N$-nitrosourea (ENU) mutagenesis screen for dominant mutations that cause neutrophilia in $G_{1}$ mice and isolated a pedigree with a mutation in NLRP1a.

\section{Results}

Mice with the mutation $N \operatorname{lrp} 1 a^{+/ Q 593 P}$ were fertile and remained healthy to at least 8 months of age, despite histological evidence of a multi-organ neutrophilic inflammatory disease characterised by meningitis, hepatitis, pneumonitis, pancreatitis, pulmonary peri-arteritis, myocarditis and inflammatory bowel disease. In Nlrp1a ${ }^{\mathrm{Q593P} / \mathrm{Q} 593 P}$ homozygotes, a similar but lethal condition developed by 3-5 months of age. Neutrophil counts in these animals were 15-fold higher than wildtype, and they exhibited lymphopenia and splenomegaly. By breeding with genetically deficient mice we showed that the lethal systemic inflammatory disease was ameliorated by removing Caspase- 1 and IL-1R but was independent of ASC. On the other hand, deletion of IL-18 increased the number of neutrophils in the blood, and greatly accelerated the onset of disease.

\section{Conclusion}

In summary we show for the first time in vivo the effect of an activating mutation in NLRP1, which causes autoinflammatory disease. We demonstrate that this disease is caused by IL- $1 \beta$ and Caspase-1, but not ASC. Surprisingly, IL-18 is beneficial for this condition, suggesting that caution should be employed when blocking IL-18 in human autoinflammatory diseases. Our results strongly suggest that mutations in human NLRP1 would cause autoinflammatory disease.

\author{
Competing interests \\ None declared. \\ Published: 8 November 2013
}

doi:10.1186/1546-0096-11-S1-A195

Cite this article as: Masters et al:: OR11-006 - A mutation in NLRP1A

causes autoinflammation. Pediatric Rheumatology 2013 11(Suppl 1):A195. 